\title{
Characterization of Wnt Signaling Genes in Diaphorina citri, Asian Citrus Psyllid
}

Chad Vosburg ${ }^{1,2}$, Max Reynolds ${ }^{1}$, Rita Noel ${ }^{1}$, Teresa Shippy ${ }^{3}$, Prashant S Hosmani ${ }^{4}$, Mirella Flores-Gonzalez ${ }^{4}$, Lukas A Mueller ${ }^{4}$, Wayne B Hunter ${ }^{5}$, Susan J Brown ${ }^{3}$, Tom D’Elia ${ }^{1}$ and Surya Saha ${ }^{4,6}$

${ }^{1}$ Indian River State College, Fort Pierce, FL 34981

2 Department of Plant Pathology and Environmental Microbiology, The Pennsylvania State University, University Park, PA 16802

${ }^{3}$ KSU Bioinformatics Center, Division of Biology, Kansas State University, Manhattan, KS

${ }^{4}$ Boyce Thompson Institute, Ithaca, NY 14853

${ }^{5}$ USDA-ARS, U.S. Horticultural Research Laboratory, Fort Pierce, FL 34945

${ }^{6}$ Animal and Comparative Biomedical Sciences, University of Arizona, Tucson, AZ 85721

\begin{abstract}
:
The Asian citrus psyllid, Diaphorina citri, is an insect vector that transmits Candidatus Liberibacter asiaticus, the causal agent of the Huanglongbing (HLB) or citrus greening disease. This disease has devastated Florida's citrus industry and threatens California's industry as well as other citrus producing regions around the world. To find novel solutions to the disease, a better understanding of the vector is needed. The $D$. citri genome has been used to identify and characterize genes involved in Wnt signaling pathways. Wnt signaling is utilized for many important biological processes in metazoans, such as patterning and tissue generation. Curation based on RNA sequencing data and sequence homology confirm twenty four Wnt signaling genes within the $D$. citri genome, including homologs for beta-catenin, Frizzled receptors, and seven Wnt-ligands. Through phylogenetic analysis, we classify $D$. citri Wnt-ligands as $W g / W n t 1, W n t 5$, Wnt6, Wnt7, Wnt10, Wnt11, and WntA. The D. citri version 3.0 genome with chromosomal length scaffolds reveals a conserved Wnt1-Wnt6-Wnt10 gene cluster with gene configuration similar to that in Drosophila melanogaster. These findings provide a greater insight into the evolutionary history of $D$. citri and Wnt signaling in this important hemipteran vector. Manual annotation was essential for identifying high quality gene models. These gene models can further be used to develop molecular systems, such as CRISPR and RNAi, that target and control D. citri populations, to manage the spread of HLB. Manual annotation of Wnt signaling pathways was done as part of a collaborative community annotation project (https://citrusgreening.org/annotation/index).
\end{abstract}

\section{Introduction:}

Diaphorina citri is the insect vector of Huanglongbing (HLB, citrus greening disease), a disease that has devastated global citrus production [1,2]. HLB management is heavily based on controlling the spread of $D$. citri. In an effort to better understand the insect's biology, the $D$. citri genome has been manually annotated to curate accurate gene model predictions. Accurate gene 
models can be used to develop novel insect control systems that utilize molecular therapeutics such as CRISPR and RNAi to control the spread of $D$. citri $[3,4]$. These molecular therapeutics would be gene-specific and reduce the reliance on broad-spectrum insecticides that have given rise to resistant $D$. citri populations [5-7].

Here, we report on $D$. citri genes involved in both canonical and noncanonical Wnt signaling. Wht signaling is important for many biological processes in metazoans such as patterning, cell polarity, tissue generation, and stem cell maintenance [8-10]. In the model insects Drosophila melanogaster and Tribolium castaneum, knockout and knockdown of Wnt ligands and other Wnt signalling components have detrimental effects on embryo development and adult homeostasis [11-15]. Wnt signaling components could therefore serve as effective knockout targets that limit the spread of D. citri, reducing HLB incidence [16].

We have curated a comprehensive repertoire of Wnt signaling genes in $D$. citri. Twentyfour gene models corresponding to canonical and noncanonical Wnt signaling genes have been annotated, including seven Wnt ligands, three frizzled homologs, arrow, armadillo/beta-catenin, and receptor tyrosine kinases ROR and doughnut. We were unable to find Wnt8/D, Wnt9, and Wnt16 as well as Wnt2-4, which have been lost in insects. The mechanisms of Wnt signaling appear to be mostly conserved and comparable to what is found in the model organism, D. melanogaster (Table 1). A model for canonical Wnt signaling in D. citri based on curated genes is shown (Figure 1). This is an important first step for understanding critical biological processes that may be targeted to control the spread of $D$. citri and may provide a broader insight into the mechanisms of Wnt signaling in this important hemipteran vector.

Table 1: Gene copy table.

\begin{tabular}{|c|c|c|c|c|c|}
\hline Gene & $\begin{array}{c}\text { Drosophila } \\
\text { melanogaster }\end{array}$ & $\begin{array}{c}\text { Apis } \\
\text { mellifera }\end{array}$ & $\begin{array}{c}\text { Tribolium } \\
\text { castaneum }\end{array}$ & $\begin{array}{c}\text { Acyrthosiphon } \\
\text { pisum }\end{array}$ & $\begin{array}{c}\text { Diaphorina } \\
\text { citri v3 }\end{array}$ \\
\hline Wnt1 & 1 & 1 & 1 & 1 & 1 \\
\hline Wnt5 & 1 & 1 & 1 & 1 & 1 \\
\hline Wnt6 & 1 & 1 & 1 & 1 & 1 \\
\hline Wnt7 & 1 & 1 & 1 & 0 & 1 \\
\hline Wnt8/D & 1 & 0 & 1 & 0 & 0 \\
\hline Wnt9 & 1 & 0 & 1 & 0 & 1 \\
\hline Wnt10 & 1 & 1 & 1 & 1 & 1 \\
\hline Wnt11 & 0 & 1 & 1 & 1 & 0 \\
\hline Wnt16 & 0 & 0 & 0 & 1 & 1 \\
\hline WntA & 0 & 1 & 1 & 2 & 1 \\
\hline pangolin & 1 & 1 & 1 & 1 & 1 \\
\hline armadillo & 1 & 1 & 2 & 1 & 1 \\
\hline wntless & 1 & 1 & 1 & 1 & 1 \\
\hline porcupine & 1 & 1 & 1 & 1 & 1 \\
\hline derailed & 2 & 1 & 0 & 1 & 1
\end{tabular}




\begin{tabular}{|c|l|l|l|l|l|}
\hline frizzled & 4 & 2 & 3 & 2 & 3 \\
\hline ROR & 2 & 2 & 3 & 2 & 2 \\
\hline dishevelled & 1 & 1 & 1 & 1 & 1 \\
\hline shaggy & 1 & 1 & 1 & 2 & 1 \\
\hline Axin & 1 & 1 & 1 & 1 & 1 \\
\hline ck1-gamma & 1 & 1 & 1 & 1 & 1 \\
\hline Apc & 2 & 1 & 1 & 1 & 1 \\
\hline
\end{tabular}

Wnt pathway ortholog numbers in five different insect species. Drosophila melanogaster, Apis mellifera, Tribolium castaneum, and Acyrthosiphon pisum copy numbers were determined using Flybase, OrthoDB, NCBI Genbank, Uniprot, and several other publications [15,17-19]. Diaphorina citri numbers represent the number of manually annotated genes in the $D$. citri v3.0 genome.

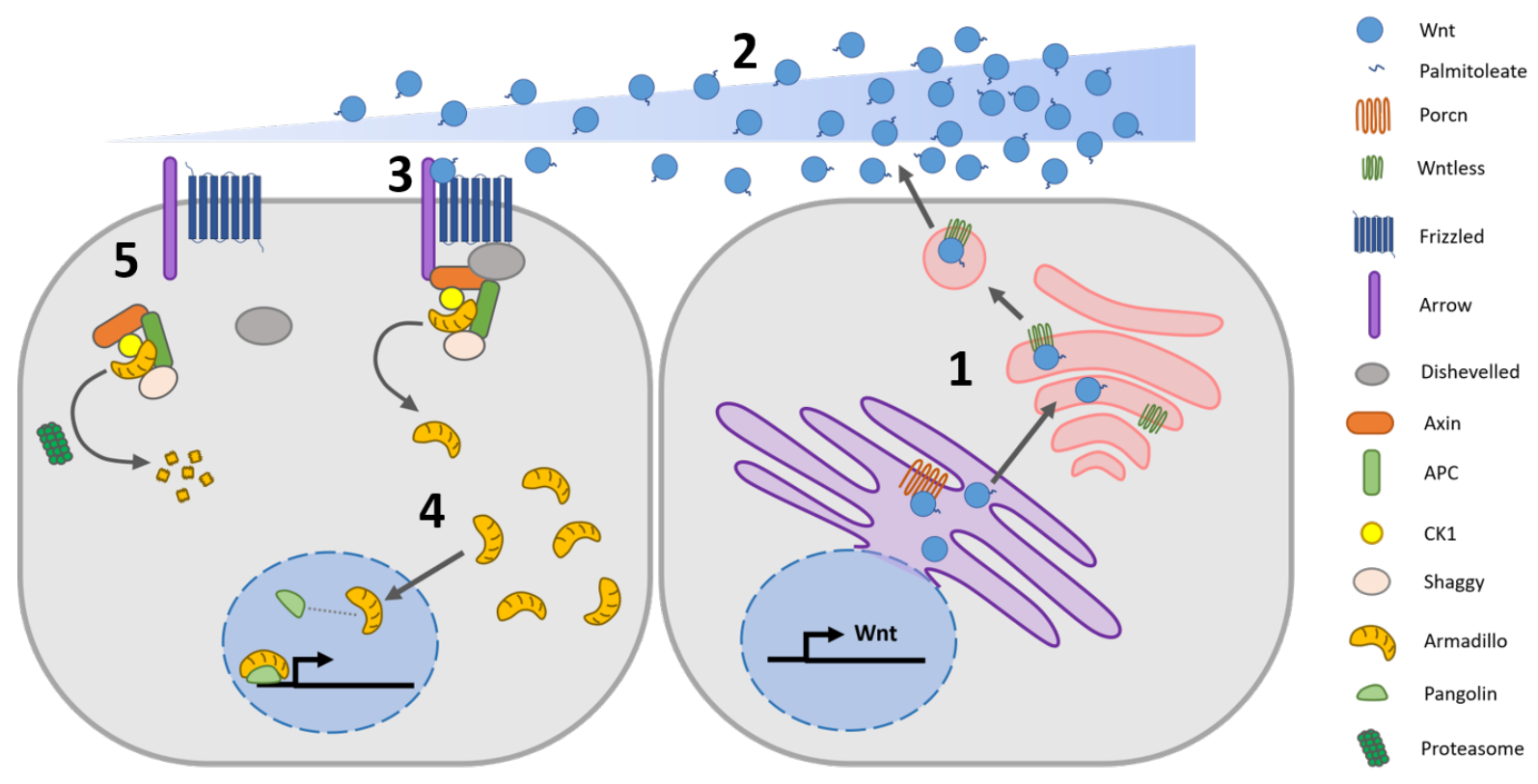

Figure 1: Theoretical model of canonical Wnt signaling cascade in $D$. citri based on curated genes. 1) Wnt is secreted. 2) Wnt concentration gradient forms. 3) Wnt binds to Frizzled and releases Armadillo. 4) Armadillo migrates into the nucleus, associates with transcription factor Pangolin, and regulates gene expression. 5) Armadillo is degraded in the absence of Wnt.

\section{Results and Discussion:}

The loss of Wnt ligand genes is more common in insects than in other metazoans [17], which leads to a highly variable array of Wnt genes and Wnt signaling components from species to species [15,18-20]. We performed a phylogenetic analysis to characterize the $D$. citri Wnt repertoire (Figure 2). Seven different $D$. citri Wnts were identified and classified as Wnt1 (also known as wingless), Wnt5, Wnt6, Wnt7, Wnt10, Wnt11, and WntA (Figure 2 and 3). In comparison, seven Wnt genes have been identified in D. melanogaster, nine in T. castaneum, and 
six in Acyrthosiphon pisum $[19,20]$. The collection of Wnt genes found in D. citri is similar to other insects, and there have been no Wnt subfamilies identified that are unique to $D$. citri. Contrary to what has been previously reported [21], D. citri does appear to possess a Wnt6 gene.

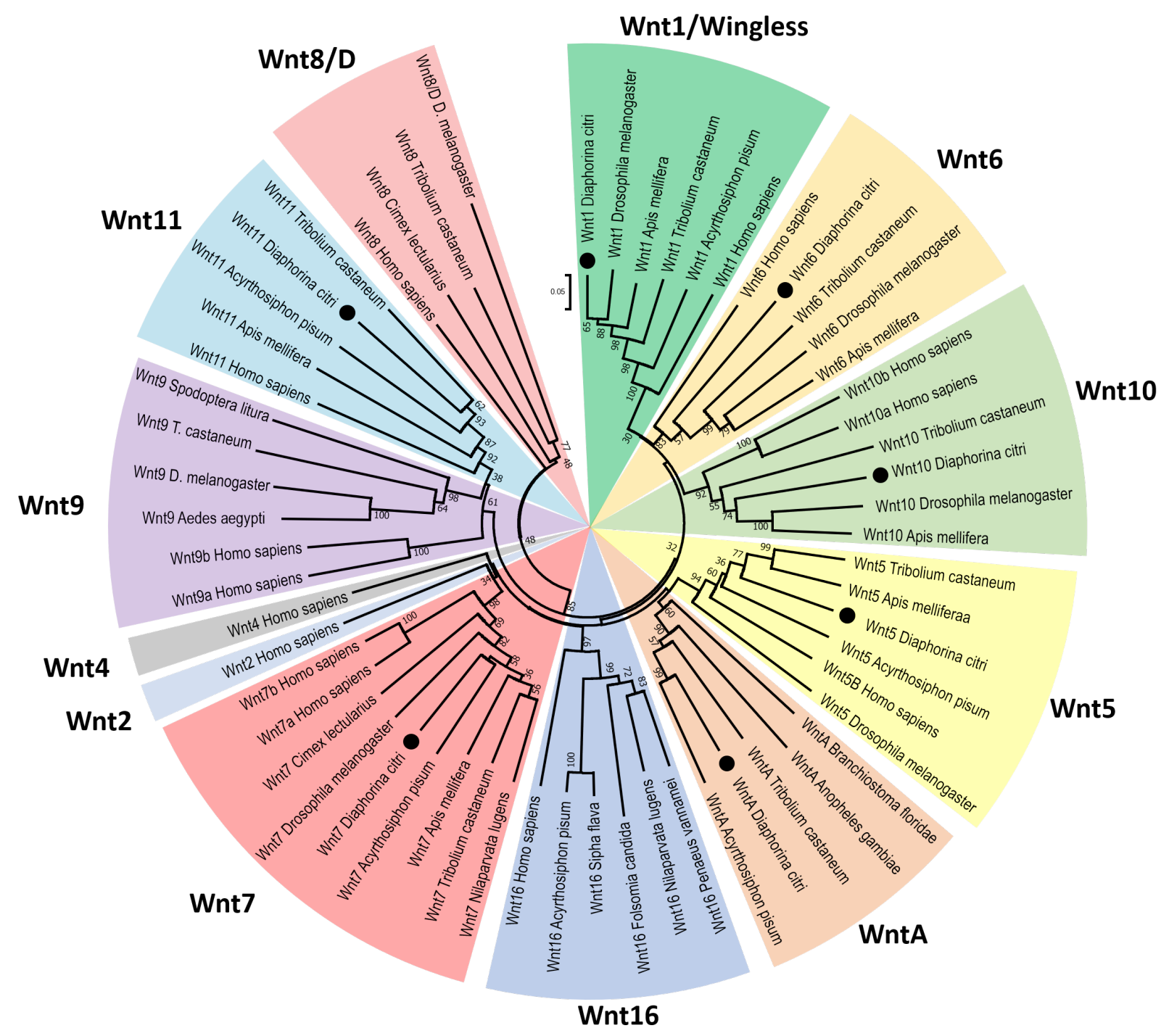

Figure 2: Neighbor-joining tree of Wnt protein sequences. Phylogenetic analysis was performed to categorize the seven $D$. citri Wnt genes (signified by dots). Wnt families are distinguished by clades and are color coded. Bootstrap values are based on 1000 replicates and values under 25 are removed. Ortholog sequences were collected from NCBI protein database (Table 3). Analysis was performed using MEGA7. 


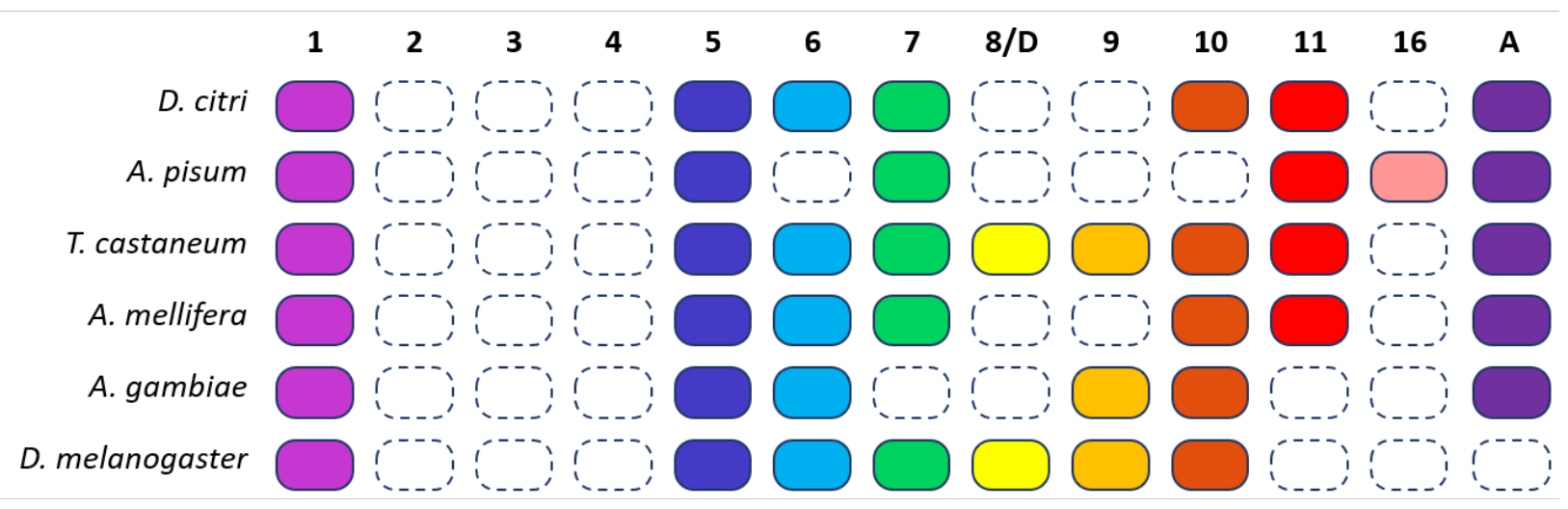

Figure 3: Wnt genes in six insects. A colored box indicates the presence of a Wnt subfamily ( 1 to 11,16 , and $A$ ) in that insect, while a white box indicates the loss of a subfamily. For example, all six species have Wnt1 and Wnt5, none have Wnt2-4, and only A. pisum has Wnt16. Homologs of Wnt8 in T. castaneum and D. melanogaster are also referred to as WntD.

Wnt1, Wnt6, and Wnt10 typically occur in very close proximity in a highly conserved gene cluster [22,23]. Accordingly, it is believed that this cluster is also conserved in $D$. citri and this notion is supported by the chromosomal length genome assembly in v3.0 [24]. The close phylogenetic relationship of Wnt1, Wnt6, and Wnt10 in D. citri (Figure 2) supports the hypothesis that this cluster is the result of an ancient duplication event, one that may predate the divergence of cnidarians and bilaterians [23]. The orientation of these clustered D. citri Wnt genes is similar to that found in $D$. melanogaster and differs from what may be a basal arthropodal organization of Wnts found in species of Coleoptera, Hymenoptera, and Cladocera (Figure 4). Wnt9 is also associated with this gene cluster when present in the genome. However, as with A. pisum, Wnt9 was not found in the $D$. citri genome and appears to have been lost during evolution. A second Wnt cluster, Wnt5 and Wnt7, is also common among non-insect metazoans. This cluster is not seen in $D$. citri despite the presence of both genes.

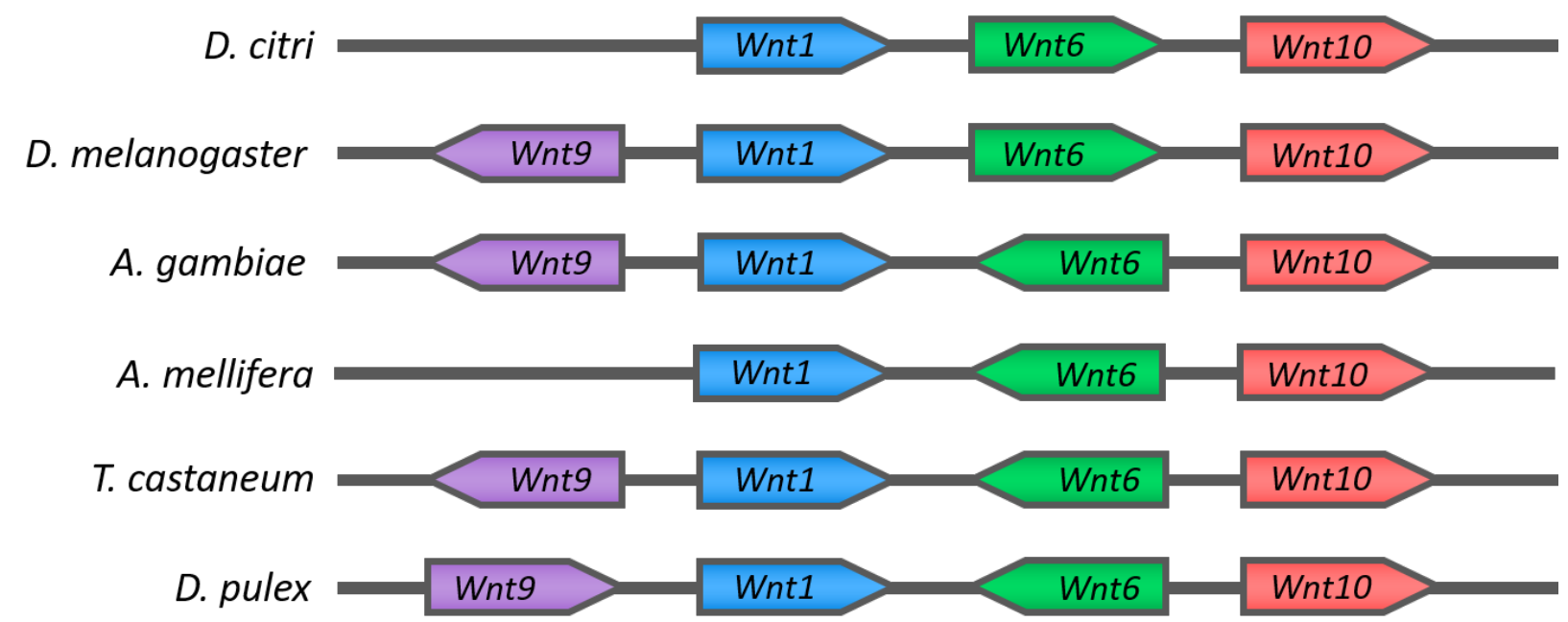

Figure 4: Wnt1-6-10 Cluster comparison. Organization of Wnt1-6-10 cluster in D. citri is similar to $D$. melanogaster and differs from what may be a basal arthropod gene arrangement seen in A. gambiae, T. castaneum, A. mellifera, and D. pulex. Gene lengths are not to scale. 
The mechanisms that act to conserve these Wnt gene clusters are not well understood. In the basal metazoan, Nematostella vectensis, clustered Wnt genes do not exhibit similar expression patterns or Hox-like collinearity [22] and may not share regulatory elements. Data obtained from the Psyllid Expression Network (PEN) [25] available on citrugreening.org shows varying levels of expression amongst the clustered genes in different life stages of $D$. citri (Figure 5). However, it appears that Wnt1 and 10 are similarly upregulated during embryonic psyllid development and downregulated during the adult stage, and similar transcript levels of Wnt 1 and 6 are seen in the nymphal stage. This suggests there may be shared regulation dependent upon life stage. Furthermore, ordering within the clusters is subject to rearrangement (Figure4)[20,22]. This may indicate that gene directionality is not a factor in conserving this cluster. Our annotation findings support the hypothesis that the Wnt1-6-10 cluster is being preserved through either natural selection or an unknown mechanism, and a better understanding of the regulatory hierarchy that controls Wnt expression might shed light on the significance of Wnt gene associations in the genome.

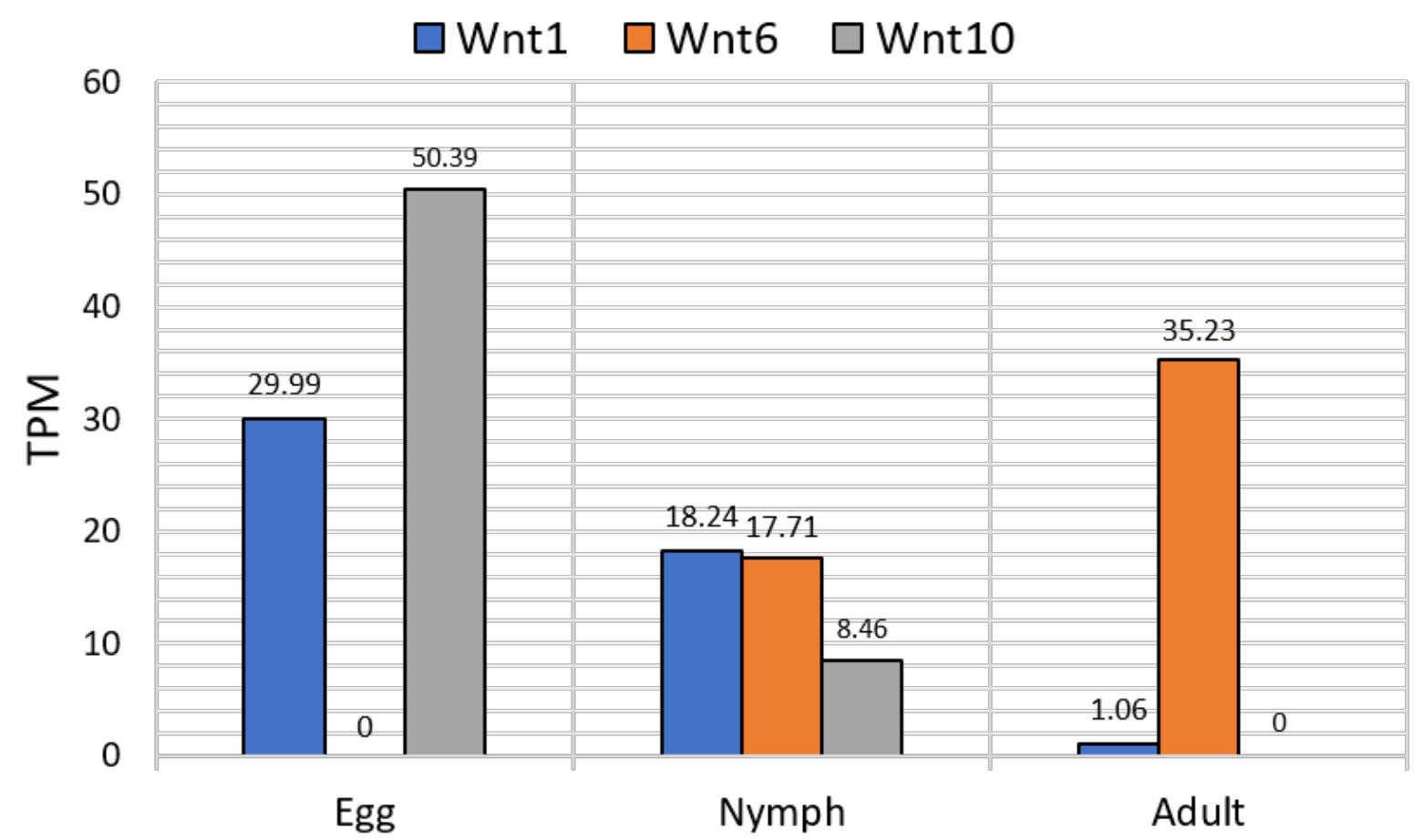

Figure 5: Transcript levels of clustered Wnt transcripts during different $\boldsymbol{D}$. citri life stages. Whole body transcript extractions were performed on egg, nymph, and adult stages. Samples were collected from Citrus macrophylla and were not infected with Candidatus Liberibacter asiaticus. RNAseq data was collected from PEN and available on citrusgreening.org. Expression values shown in transcripts per million (TPM).

The organization of the genomic reference sequence into chromosomal length scaffolds was essential for revealing $D$. citri gene clustering. The previous genome assemblies were often unsupportive in confirming the proximity of genes due to the shorter scaffold lengths. Genome v2.0 assembly errors had likely misrepresented the location of Wnt10, making it appear to be separated from Wnt1 and Wnt6. A complete Wnt1-6-10 cluster was found in the improved 
chromosome length assembly v3.0. Thus, the quality of the reference genome should be considered when performing phylogenetic studies.

Orthologs for Wnt2, Wnt3, Wnt4, Wnt8/D, Wnt9, and Wnt16 were not located in the D. citri genome. The close identity of certain Wnt subfamilies makes distinguishing between them difficult, however, the loss of Wnt2-4 is expected as they are absent in all insects [17]. Apis mellifera and the hemipteran $A$. pisum have been reported to lack $W n t 8 / D$, and perhaps this $W n t$ subfamily has been lost in the divergence from other insect groups [19]. Additionally, Wnt16 was not found in D. citri v3.0. This finding contrasts with the gene predictions of other hemipteran genomes available at NCBI, namely A. pisum, Sipha flava, and Nilaparvata lugens (Figure 2).

Based on whole body RNA extractions collected from PEN, Wnt6 has the highest average transcript levels of all the Wnt genes in both nymph and adult psyllids (Figure 6). The relatively high amount of Wnt6 transcripts suggests that it is important during both metamorphosis and adult stage homeostasis and may serve as a good knockout target for molecular therapeutics. Transcript expression of Wnt6 in adults is mainly concentrated in the legs and thorax, averaging 102 transcripts per million (TPM) and 272 TPM, respectively. This is considerably higher than all other Wnt genes in these tissues which only average between 0.26 and 3 TPM. It is unclear if other Wnts can be upregulated to compensate for the loss of Wnt6, and perhaps targeting multiple Wnt genes or the mechanisms by which Wnt is secreted (i.e. Porcupine and Wntless) would be more disruptive to $D$. citri physiology.

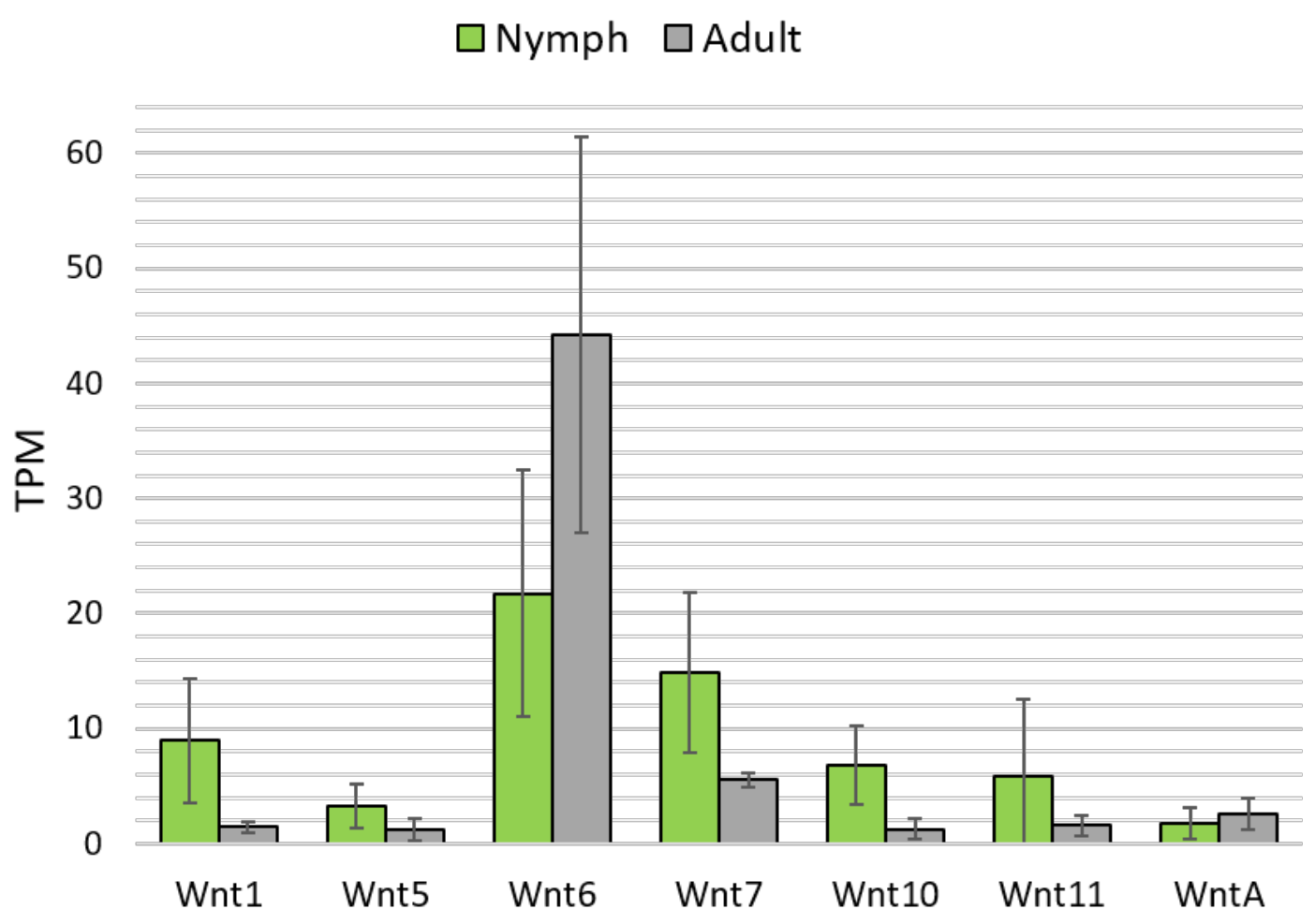

Figure 6: Transcript levels of $D$. citri Wnt repertoire in both nymph and adult psyllids from whole body RNA extractions. Green bars indicate the average transcript levels for Wnt in nymph samples, and grey bars represent the average transcript levels for Wnt in adult samples. Averages 
are based on six nymph samples and six adult samples. Expression levels shown in transcripts per million (TPM). Standard deviation of samples is shown by error bars. RNAseq data was collected from PEN and available on citrusgreening.org.

Several receptors and co-receptors associated with canonical and non-canonical signaling have been identified (Table 2). Three paralogs for the Wnt receptor encoding frizzled have been found in $D$. citri. We classified and numerically designated $D$. citri's three frizzled genes based on how their encoded protein sequences form clades with $D$. melanogaster orthologs (Figure 7). Our analysis showed that $D$. citri, and other hemipterans such as Halymorpha halys and N. lugens, possess a Frizzled protein similar to $D$. melanogaster's Frizzled 3. Some hemipteran Frizzled orthologs form a distinct clade separate from the Dipteran sequences (Figure 7). The hemipteran clade suggests that these genes could belong to a different subfamily of Frizzled, maybe one specific to Hemiptera, although this ortholog has not been reported in the $A$. pisum genome [19].

Table 2: Gene Evidence Table

\begin{tabular}{|c|c|c|c|c|c|c|}
\hline Gene & OGS Identifier & MCOT & $\begin{array}{c}\text { de novo } \\
\text { transcriptome }\end{array}$ & Iso-Seq & RNA-Seq & Ortholog \\
\hline Wnt1 & Dcitr04g11660.1.1 & $X$ & $X$ & & $X$ & $X$ \\
\hline Wnt5 & Dcitr13g03650.1.1 & $X$ & $X$ & & $X$ & \\
\hline Wnt6 & Dcitr04g11650.1.1 & $X$ & $X$ & $X$ & $X$ & \\
\hline Wnt7† & Dcitr13g03730.1.1 & $X$ & $X$ & & $X$ & $X$ \\
\hline Wnt10 & Dcitr04g11640.1.1 & $X$ & $X$ & & $X$ & $X$ \\
\hline Wnt11 & Dcitr09g05250.1.1 & $X$ & $X$ & & $X$ & \\
\hline WntA & Dcitr13g02920.1.1 & $X$ & $X$ & & $X$ & $X$ \\
\hline pangolint & Dcitr06g15680.1.1 & $x$ & $X$ & & $X$ & \\
\hline armadillo & Dcitr10g09220.1.1 & $X$ & & $X$ & $X$ & $X$ \\
\hline wntless & Dcitr01g07340.1.1 & $X$ & $X$ & $x$ & $X$ & $X$ \\
\hline porcupine & Dcitr13g04750.1.1 & $x$ & $x$ & $x$ & $X$ & \\
\hline derailed & Dcitr01g12220.1.1 & $X$ & $X$ & $X$ & $X$ & \\
\hline doughnut & Dcitr01g07650.1.1 & $X$ & $X$ & $X$ & $X$ & $X$ \\
\hline arrow & Dcitr11g02670.1.1 & $X$ & $X$ & $X$ & $X$ & $X$ \\
\hline frizzled & Dcitr04g04630.1.1 & $x$ & $X$ & & $X$ & \\
\hline frizzled 2 & Dcitr10g03570.1.1 & $x$ & $X$ & $x$ & $X$ & \\
\hline frizzled 3 & Dcitr01g12100.1.1 & $x$ & $X$ & $X$ & & \\
\hline ROR1 & $\begin{array}{l}\text { Dcitr05g14430.1.1 } \\
\text { Dcitr05g14430.1.2 }\end{array}$ & $x$ & $x$ & $X$ & $X$ & $X$ \\
\hline$R O R 2$ & Dcitr08g10450.1.1 & $X$ & $X$ & $X$ & $X$ & $X$ \\
\hline dishevelled & Dcitr01g03830.1.1 & $X$ & $X$ & & $X$ & $X$ \\
\hline shaggy & Dcitr03g15060.1.1 & $X$ & $X$ & $X$ & $X$ & $X$ \\
\hline Axin & Dcitr07g09620.1.1 & $X$ & $X$ & & $X$ & \\
\hline ck1-gamma & Dcitr11g04200.1.1 & $X$ & $X$ & $X$ & $X$ & $X$ \\
\hline Apc-like & Dcitr07g12790.1.1 & $X$ & $X$ & & $X$ & \\
\hline
\end{tabular}


† Gene is manually annotated as a partial model in Genome v3.0. A complete representation of the gene and protein sequence can be determined from MCOT transcriptome data.

Table of evidence supporting gene annotation. Manually annotated Wnt pathway genes in Diaphorina citri. Number of isoforms is noted in parentheses if there are more than one. There are 24 gene models in total. Each gene model has been assigned an identifier, and the evidence used to validate or modify the structure of the gene model has been listed. The table is marked with an ' $\mathrm{X}$ ' when supporting evidence of MCOT, de novo transcriptome, Iso-Seq, RNA-Seq and ortholog support is present. MCOT: comprehensive transcriptome based on genome MAKER, Cufflinks, Oasis, and Trinity transcript predictions; MAKER: gene predictions; De novo transcriptome: an independent transcriptome using Iso-Seq long-reads and RNA-Seq data; IsoSeq transcripts: full-length transcripts generated with Pacific Biosciences technology; RNA-Seq: reads mapped to genome are also used as supporting evidence for splice junctions; Ortholog evidence: proteins from related hemipteran species and Drosophila melanogaster.

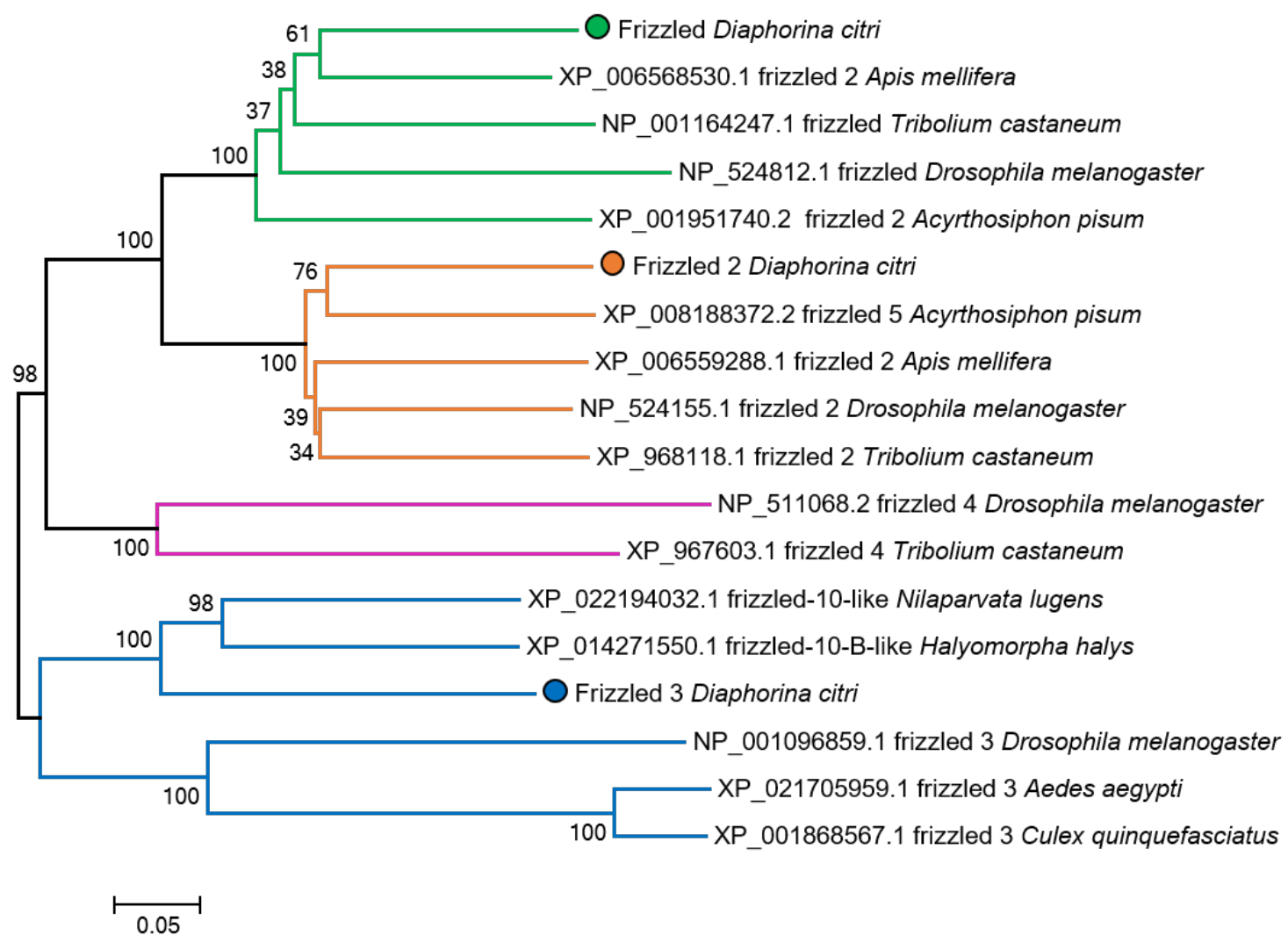

Figure 7: Neighbor-joining tree of insect Frizzled protein sequences. Proteins grouped in the Frizzled 1 subfamily are highlighted in green, Frizzled 2 in orange, Frizzled 3 in blue, and Frizzled 4 in magenta. Circles indicate the $D$. citri sequences. Some NCBI sequences (such as XP_006568530.1, XP_008188372.2, and XP_022194032.1) may have numeric labels derived from computational predictions that do not reflect sequence or functional similarity. Analysis performed using MEGA7. 
Orthologs for both ROR1 and ROR2 have been identified. Interestingly, ROR1 has two isoforms, the first of which contains an immunoglobulin (IG) domain that is lacking from isoform 2 (Figure 8). ROR1 isoform 2 (Dcitr05g14430.1.2) appears to average higher transcript levels in $D$. citri egg, nymph, and adult tissues than ROR1 isoform 1 (Dcitr05g14430.1.1) based on PEN data (Figure 9). A large number of transcripts for isoform 2 were detected in the psyllid egg (Figure 9). This suggests that expression of isoform 2 may have an important role in the early developmental stages of $D$. citri.

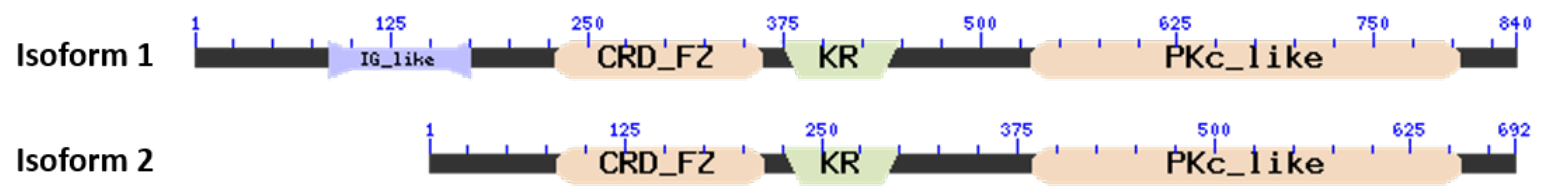

Figure 8: Domain comparison of ROR1 isoforms. The immunoglobulin domain (IG_like) is present in isoform 1. Other shared domains include a cysteine-rich frizzled domain (CRD_FZ), a Kringle domain (KR), and a protein kinase catalytic domain (PKc_like). Domains were calculated and visualized using NCBI's Conserved Domain Architecture Retrieval Tool (CDART).

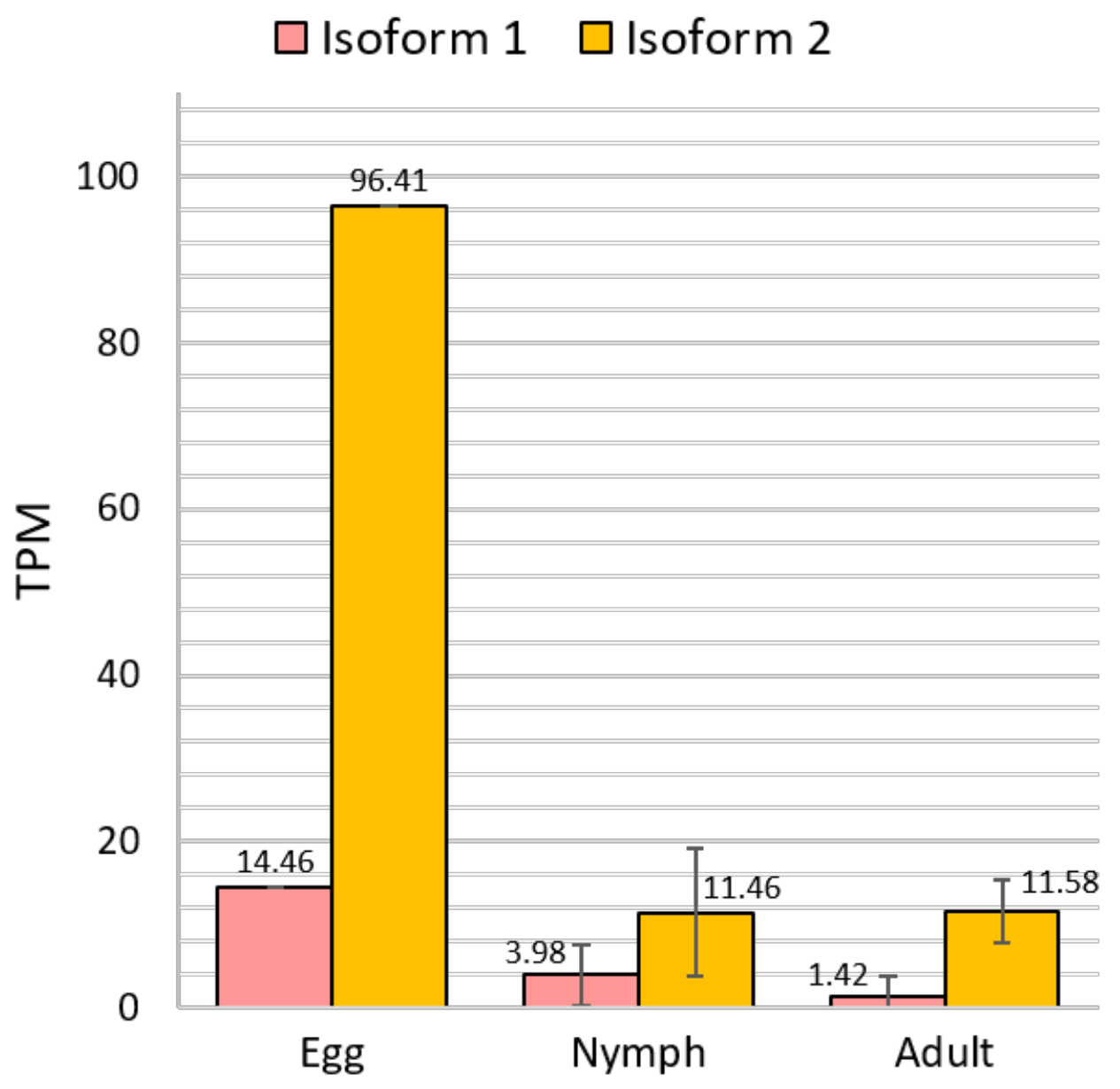

Figure 9: Expression of ROR1 Isoforms in egg, nymph and adult $D$. citri. Blue bars indicate the average transcript levels for isoform 1 (Dcitr05g14430.1.1), and orange bars indicate the average 
transcript levels for isoform 2 (Dcitr05g14430.1.2). Note: only one egg sample was used for comparison. Egg transcripts extracted from the whole egg (1 sample total), Nymph transcripts extracted from the full body (six samples total), and adult transcripts extracted from the full body, abdomen, and thorax (14 samples total). Expression values shown in transcripts per million (TPM). Data labels note the average TPM. Standard deviation of samples is shown by error bars. RNAseq data was collected from PEN and available at citrusgreening.org.

\section{Conclusion:}

Controlling the spread of $D$. citri is an important strategy for reducing the spread of HLB. With this study we hope to provide a greater insight into $D$. citri biology as well as accurate gene models that can be used in future research and applications. We have curated a comprehensive repertoire of Wnt signaling genes in D. citri. In total, 24 gene models corresponding to canonical and noncanonical Wnt signaling have been annotated. The mechanisms of Wnt signaling appear to be mostly conserved and comparable to that which is found in D. melanogaster and other insects. These findings provide a greater insight into the evolutionary history of $D$. citri and Wnt signaling in this important hemipteran vector. Manual annotation and an improved genome assembly with chromosomal length scaffold were essential for identifying high quality gene models. Future work could utilize these gene models in developing CRISPR and RNAi systems that target and disrupt critical biological processes in D. citri, thus controlling the spread of HLB. This work was done as part of a collaborative community annotation project (https://citrusgreening.org/annotation/index).

\section{Methods:}

A complete detail of the methodology used is available at https://www.protocols.io/private/9207DE0C0FD911EBB41C0A58A9FEAC2A. To summarize, orthologous protein sequences for Wnt pathway genes were collected from the NCBI protein database and used to BLAST search the D. citri MCOT transcriptome database available on citrusgreening.org. The MCOT transcriptome is a transcriptome assembly utilizing Maker, Cufflinks, Oasis, and Trinity pipelines to provide a comprehensive set of predicted gene models. High scoring MCOT models were then searched on the NCBI protein database using NCBI BLAST to confirm the viability of the predicted MCOT models. The high scoring MCOT models that had promising $\mathrm{NCBI}$ search results were used to search the $D$. citri assembled genome. Genome regions of high sequence identity to the query sequence were investigated within JBrowse. Gene models were manually annotated using the Apollo application of JBrowse, utilizing mapped DNASeq, RNA-Seq, Iso-Seq, ortholog data, and other lines of evidence to edit and confirm manual annotations and gene structure. The gene models were analyzed with NCBI BLAST to assess their completeness. MUSCLE multiple sequence alignments of the $D$. citri gene model sequences and orthologous sequences were created through MEGA7 [26]. Neighbor-joining trees were constructed using MEGA7 with p-distance for determining branch length and one thousand bootstrapping replications to measure the precision of branch placement. In special cases, 
phylogenetic analysis in conjunction with NCBI BLAST scores was used to properly name and characterize the manually annotated gene models.

Table 3: Accessions for Wnt phylogenetic tree

\begin{tabular}{|c|c|c|c|}
\hline NCBI Accession: & Species: & NCBI Protein Name: & Referred to In Fig. 2 as: \\
\hline XP_002609873.1 & Branchiostoma floridae & $\begin{array}{l}\text { hypothetical protein } \\
\text { BRAFLDRAFT_60204 }\end{array}$ & WntA \\
\hline XP_024085687.1 & Cimex lectularius & Wnt-8b-like & Wnt8 \\
\hline XP_014257242.2 & Cimex lectularius & Wnt-7b isoform $X 1$ & Wnt7 \\
\hline NP_476972.2 & $\begin{array}{l}\text { Drosophila } \\
\text { melanogaster }\end{array}$ & $\begin{array}{l}\text { Wnt oncogene analog } 4 \\
\text { isoform } \mathrm{A}\end{array}$ & Wnt9 \\
\hline NP_476924.1 & $\begin{array}{l}\text { Drosophila } \\
\text { melanogaster }\end{array}$ & $\begin{array}{l}\text { Wnt oncogene analog } 5 \\
\text { isoform A }\end{array}$ & Wnt5 \\
\hline NP_476810.1 & $\begin{array}{l}\text { Drosophila } \\
\text { melanogaster }\end{array}$ & $\begin{array}{l}\text { Wnt oncogene analog } 2 \\
\text { isoform A }\end{array}$ & Wnt7 \\
\hline NP_609109.3 & $\begin{array}{l}\text { Drosophila } \\
\text { melanogaster }\end{array}$ & $\begin{array}{l}\text { Wnt oncogene analog } \\
10\end{array}$ & Wnt10 \\
\hline NP_609108.3 & $\begin{array}{l}\text { Drosophila } \\
\text { melanogaster }\end{array}$ & $\begin{array}{l}\text { Wnt oncogene analog } 6 \\
\text { isoform B }\end{array}$ & Wnt6 \\
\hline NP_523502.1 & $\begin{array}{l}\text { Drosophila } \\
\text { melanogaster }\end{array}$ & Wingless & Wnt1 \\
\hline NP_650272.1 & $\begin{array}{l}\text { Drosophila } \\
\text { melanogaster }\end{array}$ & wnt inhibitor of dorsal & Wnt8/D \\
\hline ALO81632.1 & Penaeus vannamei & Wnt-16 & Wnt16 \\
\hline OXA45577.1 & Folsomia candida & Wnt-16 & Wnt16 \\
\hline XP_025422997.1 & Sipha flava & Wnt-16-like & Wnt16 \\
\hline
\end{tabular}




\begin{tabular}{|c|c|c|c|}
\hline XP_022821085.1 & Spodoptera litura & Wnt-4-like & Wnt9 \\
\hline XP_015835609.1 & Tribolium castaneum & Wnt-4 & Wnt9 \\
\hline XP_008196351.1 & Tribolium castaneum & Wnt-7b isoform X1 & Wnt7 \\
\hline XP_008195370.1 & Tribolium castaneum & Wnt-1 & WntA \\
\hline XP_015835988.1 & Tribolium castaneum & Wnt-11b-1 isoform X1 & Wnt11 \\
\hline XP_008193179.1 & Tribolium castaneum & Wnt-10a isoform X1 & Wnt10 \\
\hline NP_001164137.1 & Tribolium castaneum & Wnt6 protein precursor & Wnt6 \\
\hline NP_001107822.1 & Tribolium castaneum & wingless precursor & Wnt1 \\
\hline XP_974684.1 & Tribolium castaneum & Wnt-5b & Wnt5 \\
\hline XP_971439.1 & Tribolium castaneum & Wnt-8a isoform X1 & Wnt8 \\
\hline XP_021702998.1 & Aedes aegypti & Wnt-4 & WntA \\
\hline XP_557821.3 & Anopheles gambiae & AGAP008678-PA & WntA \\
\hline XP_006561993.1 & Apis mellifera & Wnt-5b isoform X1 & Wnt5 \\
\hline XP_006557287.1 & Apis mellifera & Wnt-7b isoform $X 1$ & Wnt7 \\
\hline XP_006567803.2 & Apis mellifera & Wnt-11b & Wnt11 \\
\hline XP_016771882.1 & Apis mellifera & Wnt-6 isoform X1 & Wnt6 \\
\hline XP_026300091.1 & Apis mellifera & Wnt-1 & Wnt1 \\
\hline XP_396944.4 & Apis mellifera & Wnt-10b & Wnt10 \\
\hline XP_001949667.2 & Acyrthosiphon pisum & Wnt-5b & Wnt5 \\
\hline XP_016664156.1 & Acyrthosiphon pisum & Wnt-16 & Wnt16 \\
\hline XP_001948541.2 & Acyrthosiphon pisum & Wnt-2 & Wnt7 \\
\hline XP_001947400.1 & Acyrthosiphon pisum & Wnt-1 & WntA \\
\hline XP_001944637.3 & Acyrthosiphon pisum & $\begin{array}{l}\text { Wnt-11b-like isoform } \\
\mathrm{X} 1\end{array}$ & Wnt11 \\
\hline XP_001945295.1 & Acyrthosiphon pisum & Wnt-1 & Wnt1 \\
\hline
\end{tabular}




\begin{tabular}{|l|l|l|l|}
\hline XP_022184533.1 & Nilaparvata lugens & Wnt-16-like & Wnt16 \\
\hline XP_022188550.1 & Nilaparvata lugens & Wnt-7b & Wnt7 \\
\hline BAB62039.1 & Homo sapiens & WNT5B & Wnt5B \\
\hline NP_003382.1 & Homo sapiens & Wnt-2 precursor & Wnt2 \\
\hline NP_057171.2 & Homo sapiens & Wnt-16 isoform 2 & Wnt16 \\
\hline NP_004616.2 & Homo sapiens & Wnt-7a precursor & Wnt7a \\
\hline NP_478679.1 & Homo sapiens & Wnt-7b precursor & Wnt7b \\
\hline NP_004617.2 & Homo sapiens & Wnt-11 precursor & Wnt11 \\
\hline NP_003386.1 & Homo sapiens & Wnt-9a precursor & Wnt9a \\
\hline NP_003387.1 & Homo sapiens & $\begin{array}{l}\text { Wnt-9b isoform 1 } \\
\text { precursor }\end{array}$ & Wnt9b \\
\hline NP_110388.2 & Homo sapiens & Wnt-4 precursor & Wnt4 \\
\hline NP_079492.2 & Homo sapiens & Wnt-10a precursor & Wnt10a \\
\hline NP_003385.2 & Homo sapiens & Wnt-10b precursor & Wnt10b \\
\hline NP_006513.1 & Homo sapiens & Wnt-6 precursor & Wnt6 \\
\hline NP_005421.1 & Homo sapiens & $\begin{array}{l}\text { proto-oncogene Wnt-1 } \\
\text { precursor }\end{array}$ & Wnt1 \\
\hline NP_001287867.1 & Homo sapiens & $\begin{array}{l}\text { Wnt-8a isoform 1 } \\
\text { precursor }\end{array}$ & Wnt8 \\
\hline
\end{tabular}

Acknowledgements: This work was supported by USDA-NIFA grant 2015-70016-23028. We thank Alistair McGregor for valuable discussion.

\section{References}

1. Bové JM. Huanglongbing: A destructive, newly-emerging, century-old disease of citrus. J Plant Pathol. Società Italiana di Patologia Vegetale (SIPaV); 2006;88:7-37.

2. Gottwald TR. Current epidemiological understanding of citrus Huanglongbing. Annu Rev Phytopathol. 2010;48:119-39.

3. Hunter WB, Gonzalez MT, Tomich J. BAPC-assisted CRISPR/Cas9 System: Targeted Delivery into Adult Ovaries for Heritable Germline Gene Editing (Arthropoda: Hemiptera) [Internet]. Cold 
Spring Harbor Laboratory. 2018 [cited 2020 Nov 23]. p. 478743.

https://www.biorxiv.org/content/10.1101/478743v1.abstract

4. Hunter WB, Clarke S-KV, Mojica AFS, Paris TM, Miles G, Metz JL, et al. Advances in RNA suppression of the Asian citrus psyllid vector and bacteria (huanglongbing pathosystem). Asian Citrus Psyllid: Biology, Ecology and Management of the Huanglongbing Vector. CABI; 2020;258.

5. Chen XD, Stelinski LL. Resistance Management for Asian Citrus Psyllid, Diaphorina citri Kuwayama, in Florida. Insects [Internet]. 2017;8. http://dx.doi.org/10.3390/insects8030103

6. Kanga LHB, Eason J, Haseeb M, Qureshi J, Stansly P. Monitoring for Insecticide Resistance in Asian Citrus Psyllid (Hemiptera: Psyllidae) Populations in Florida. J Econ Entomol. 2016;109:832-6.

7. Pardo S, Martínez AM, Figueroa JI, Chavarrieta JM, Viñuela E, Rebollar-Alviter Á, et al. Insecticide resistance of adults and nymphs of Asian citrus psyllid populations from Apatzingán Valley, Mexico. Pest Manag Sci. 2018;74:135-40.

8. Holstein TW. The evolution of the Wnt pathway. Cold Spring Harb Perspect Biol. 2012;4:a007922.

9. Nusse R, Fuerer C, Ching W, Harnish K, Logan C, Zeng A, et al. Wnt signaling and stem cell control. Cold Spring Harb Symp Quant Biol. 2008;73:59-66.

10. Nusse R. The Wnt gene homepage. c2020 [cited 2020 Feb 3].

http://web.stanford.edu/group/nusselab/cgi-bin/wnt/

11. Klingensmith J, Nusse R. Signaling by wingless in Drosophila. Dev Biol. 1994;166:396-414.

12. Bhanot P, Fish M, Jemison JA, Nusse R, Nathans J, Cadigan KM. Frizzled and Dfrizzled-2 function as redundant receptors for Wingless during Drosophila embryonic development. Development. The Company of Biologists Ltd; 1999;126:4175-86.

13. Kadowaki T, Wilder E, Klingensmith J, Zachary K, Perrimon N. The segment polarity gene porcupine encodes a putative multitransmembrane protein involved in Wingless processing. Genes Dev. 1996;10:3116-28.

14. Bolognesi R, Farzana L, Fischer TD, Brown SJ. Multiple Wnt genes are required for segmentation in the short-germ embryo of Tribolium castaneum. Curr Biol. 2008;18:1624-9.

15. Beermann A, Prühs R, Lutz R, Schröder R. A context-dependent combination of Wnt receptors controls axis elongation and leg development in a short germ insect. Development. 2011;138:2793-805.

16. Suzuki T, Trush O, Yasugi T, Takayama R, Sato M. Wnt Signaling Specifies Anteroposterior Progenitor Zone Identity in the Drosophila Visual Center. J Neurosci. 2016;36:6503-13. 
17. Janssen R, Le Gouar M, Pechmann M, Poulin F, Bolognesi R, Schwager EE, et al. Conservation, loss, and redeployment of Wnt ligands in protostomes: implications for understanding the evolution of segment formation [Internet]. BMC Evolutionary Biology. 2010. p. 374. http://dx.doi.org/10.1186/1471-2148-10-374

18. Bao R, Fischer T, Bolognesi R, Brown SJ, Friedrich M. Parallel duplication and partial subfunctionalization of $\beta$-catenin/armadillo during insect evolution. Mol Biol Evol. 2012;29:647-62.

19. Shigenobu S, Bickel RD, Brisson JA, Butts T, Chang C-C, Christiaens O, et al. Comprehensive survey of developmental genes in the pea aphid, Acyrthosiphon pisum: frequent lineage-specific duplications and losses of developmental genes. Insect Mol Biol. Wiley Online Library; 2010;19:47-62.

20. Bolognesi R, Beermann A, Farzana L, Wittkopp N, Lutz R, Balavoine G, et al. Tribolium Wnts: evidence for a larger repertoire in insects with overlapping expression patterns that suggest multiple redundant functions in embryogenesis. Dev Genes Evol. 2008;218:193-202.

21. Doumpas N, Jékely G, Teleman AA. Wnt6 is required for maxillary palp formation in Drosophila. BMC Biol. 2013;11:104.

22. Sullivan JC, Ryan JF, Mullikin JC, Finnerty JR. Conserved and novel Wnt clusters in the basal eumetazoan Nematostella vectensis. Dev Genes Evol. 2007;217:235-9.

23. Nusse R. An ancient cluster of Wnt paralogues [Internet]. Trends in Genetics. 2001. p. 443. http://dx.doi.org/10.1016/s0168-9525(01)02349-6

24. Hosmani PS, Flores-Gonzalez M, Shippy T, Vosburg C, Massimino C, Tank W, et al.

Chromosomal length reference assembly for Diaphorina citri using single-molecule sequencing and $\mathrm{Hi}-\mathrm{C}$ proximity ligation with manually curated genes in developmental, structural and immune pathways. bioRxiv. Cold Spring Harbor Laboratory; 2019;869685.

25. Flores-Gonzalez M, Hosmani PS, Fernandez-Pozo N, Mann M, Humann JL, Main D, et al. Citrusgreening.org: An open access and integrated systems biology portal for the Huanglongbing (HLB) disease complex [Internet]. bioRxiv. 2019 [cited 2020 Jan 28]. p. 868364. https://www.biorxiv.org/content/10.1101/868364v1.abstract

26. Kumar S, Stecher G, Tamura K. MEGA7: Molecular Evolutionary Genetics Analysis Version 7.0 for Bigger Datasets. Mol Biol Evol. 2016;33:1870-4. 